\title{
Gauging hadronic systems
}

\author{
A. N. Kvinikhidze* and B. Blankleider ${ }^{\dagger}$ \\ Department of Physics, The Flinders University of South Australia, \\ Bedford Park, SA 5042, Australia
}

\begin{abstract}
We present a general method for incorporating the electromagnetic interaction into descriptions of hadronic processes based on four-dimensional scattering integral equations. The method involves the idea of gauging the scattering equations themselves, and results in electromagnetic amplitudes where an external photon is effectively coupled to every part of every strong interaction diagram in the model. Current conservation is therefore implemented in the theoretically correct fashion. To illustrate our gauging procedure we apply it to the three-nucleon problem whose strong interactions are described by standard three-body integral equations. In this way we obtain the expressions needed to calculate all possible electromagnetic processes of the three-nucleon system: the electromagnetic form factors of the three-body bound state, $p d \rightarrow p d \gamma, \gamma^{3} \mathrm{He} \rightarrow p d, \gamma^{3} \mathrm{He} \rightarrow p p n$, etc. As the photon is coupled everywhere in the strong interaction model, a unified description of the $N N N-\gamma N N N$ system is obtained. An interesting aspect of our results is the natural appearance of a subtraction term needed to avoid the overcounting of diagrams.
\end{abstract}

\section{INTRODUCTION}

There is currently great interest in the use of photons and electrons to probe the structure of hadronic systems. As the strong interactions of hadrons are often described through the solution of integral equations, there is an outstanding problem of how to extend such nonperturbative descriptions to the electromagnetic sector. An essential requirement of such an extension is that of electromagnetic current conservation.

Here we would like to present a simple but general method for constructing conserved currents that is applicable to any hadronic process defined by relativistic four-dimensional scattering equations. The method involves a direct gauging of the equations themselves and results in the photon being effectively coupled everywhere in the strong interaction model, so that current conservation is guaranteed.

To illustrate our gauging procedure, we first apply it to the two-body problem, and then to the considerably more complicated case of the relativistic three-body problem. In gauging the three-body case there exists an overcounting problem which has previously been overlooked. It is a feature of our gauging method that it solves this overcounting problem in

* On leave from The Mathematical Institute of Georgian Academy of Sciences, Tbilisi, Georgia.

†The authors would like to thank the Australian Research Council for their financial support. 
an automatic way. We then apply our results to the three-nucleon system where the further requirement of antisymmetry is fully taken into account.

Although the power of the gauging of equations method is thus well demonstrated on the example of the three-nucleon system, it should be emphasised that the same method is easily applied to systems where the number of hadrons is not conserved. Indeed we have recently used this method to gauge the the $N N-\pi N N$ system [1] which has overcounting problems all of its own even before the attachment of photons. The same method can also be used to generate three-dimensional descriptions of gauged hadronic systems by applying it to the corresponding spectator equations [2]. In this respect we have recently solved the long-standing problem of how to describe the gauged three-nucleon system within the spectator approach [3].

Finally, it is important to note that although we are concerned in this paper with the electromagnetic interaction for which current conservation is a major issue, the gauging of equations method itself is totally independent of the type of external field involved. Thus the results of this paper hold also for the case of a weak probe for which current is not conserved (of course the gauged inputs would need to be chosen appropriately).

\section{GAUGING TWO PARTICLES}

\section{A. Gauging the two-body Green function}

We consider the scattering of two distinguishable hadrons described by the Green function $G$. Then the corresponding quantity with a "photon attached" is given by the five-point function $G^{\mu}$ (see for example Ref. [5]). If $G$ and $G^{\mu}$ are exact, as specified by their definitions in field theory, then they satisfy the Ward-Takahashi (WT) identity «4.5 from which current conservation follows.

Here we demonstrate how to find $G^{\mu}$ when $G$ is given only within a particular model. Moreover, we show how to attach photons everywhere to $G$ so that the WT identity is still satisfied. We start by expressing $G$ in terms of its fully disconnected part, $G_{0}$, and the model-dependent kernel $V$ :

$$
G=G_{0}+G_{0} V G
$$

This is a symbolic equation representing, in the case of two-particle scattering, a fourdimensional integral equation. Eq. (11) is basically a topological statement regarding the two-particle irreducible structure of Feynman diagrams belonging to $G$; as such, it can be utilised directly to express the structure of the same Feynman diagrams, but with photons attached everywhere. Thus from Eq. (1) it immediately follows that

$$
G^{\mu}=G_{0}^{\mu}+G_{0}^{\mu} V G+G_{0} V^{\mu} G+G_{0} V G^{\mu} .
$$

This result expresses $G^{\mu}$ in terms of an integral equation, and illustrates what we mean by gauging an equation, in this case the gauging of Eq. (1). Implied in Eq. (2) is the result

$$
\left[G_{0} V G\right]^{\mu}=G_{0}^{\mu} V G+G_{0} V^{\mu} G+G_{0} V G^{\mu}
$$


which illustrates a rule for the gauging of products that is rather reminiscent of the product rule for derivatives. Although Eq. (3) follows from a topological argument, we shall postulate such a product rule to hold also in other cases where topological arguments may not be applicable. In Eq. (2), both $G^{\mu}$ and $G_{0}^{\mu}$ are obtained from the Green functions $G$ and $G_{0}$, respectively, by attaching photons everywhere. The gauged potential $V^{\mu}$ is similarly obtained from $V$, but with no photons attached to external legs since such contributions are already taken into account in the terms $G_{0}^{\mu} V G$ and $G_{0} V G^{\mu}$. Eq. (2) is now easily solved algebraically giving

$$
G^{\mu}=G \Gamma^{\mu} G
$$

with the electromagnetic vertex function $\Gamma^{\mu}$ being given by

$$
\Gamma^{\mu}=\Gamma_{0}^{\mu}+V^{\mu} \quad ; \quad \Gamma_{0}^{\mu}=G_{0}^{-1} G_{0}^{\mu} G_{0}^{-1} .
$$

For a two-particle system $G_{0}=d_{1} d_{2}$ so that $\Gamma_{0}^{\mu}=\Gamma_{1}^{\mu} d_{2}^{-1}+d_{1}^{-1} \Gamma_{2}^{\mu}$ where $d_{i}(i=1,2)$ is the Feynman propagator of particle $i$ and $\Gamma_{i}^{\mu}$ is the one-particle electromagnetic vertex function defined by $d_{i}^{\mu}=d_{i} \Gamma_{i}^{\mu} d_{i}$. $\Gamma_{0}^{\mu}$ is thus the sum of single nucleon currents, and $V^{\mu}$ is the twobody interaction current of the given model. For the case of two particles, this result agrees with the one of Gross and Riska [6] who used a different method, that of analysing Feynman diagram contributions, to derive this result. It is easy to show explicitly that the $G^{\mu}$ as specified by Eqs. (四) and (司) satisfies the WT identity.

\section{B. Gauging the two-body bound state wave function}

So far we have defined "gauging" to be the process where photons are attached to all places in perturbation diagrams. As Green functions and potentials have a diagrammatic interpretation, the gauging of these quantities has therefore a clear meaning. On the other hand, the bound state wave function is a purely nonperturbative quantity, and thus cannot be associated with diagrams. Nevertheless, we can define the gauged bound state wave function by formally gauging the bound-state Bethe-Salpeter equation using our product rule. Thus the gauging of the bound state equation $\psi=G_{0} V \psi$ is postulated to give $\psi^{\mu}=\left(G_{0} V\right)^{\mu} \psi+G_{0} V \psi^{\mu}$. Simple algebra then gives the explicit expression for $\psi^{\mu}$ :

$$
\psi^{\mu}=G \Gamma^{\mu} \psi .
$$

As Eq. (6) can also be obtained from the $G^{\mu}$ of Eq. (4) by taking the right hand residue at the two-body bound state pole, we see that $G_{0}^{-1} \psi^{\mu}$ is just the transition amplitude for the photo/electro-disintegration of the hadronic bound state. The bound state vertex function $\phi$, defined by $\psi=G_{0} \phi$, may be gauged in the same way. Interestingly, in contrast to $G_{0}^{-1} \psi^{\mu}$ on mass shell, neither $\psi^{\mu}$ nor $\phi^{\mu}$ conserve current.

\section{GAUGING THREE PARTICLES}




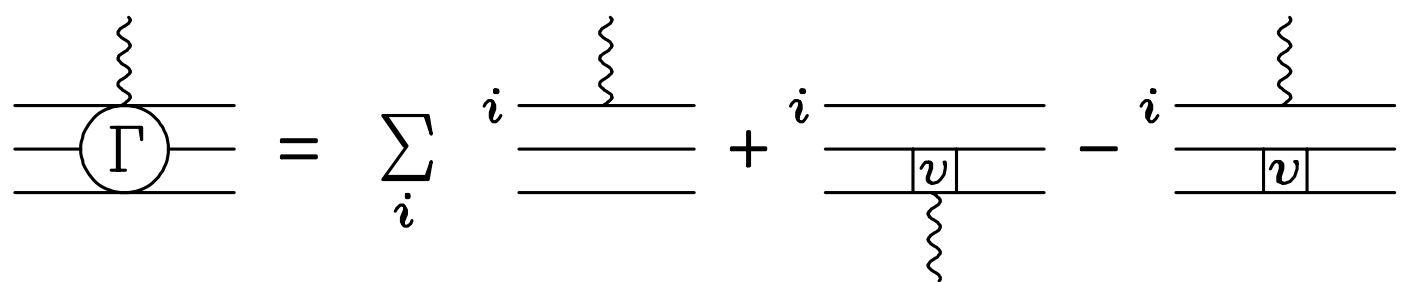

FIG. 1. Illustration of Eq. (8) expressing the three-particle electromagnetic vertex function $\Gamma^{\mu}$ as a sum of one- and two-nucleon currents.

\section{A. Gauging the three-body Green function}

It is clear that the derivation of Eqs. (四) and (5) does not depend on the number of particles. It therefore applies when $G$ is the Green function for three particles and $G^{\mu}$ is the corresponding seven-point function. In this case however $\Gamma_{0}^{\mu}=\sum_{i=1}^{3} \Gamma_{i}^{\mu} D_{0 i}^{-1}, D_{0 i}=d_{j} d_{k}$, and $V^{\mu}$ refers to the gauged three-body potential. In the absence of three-body forces, $V$ is a sum of three disconnected potentials: $V=V_{1}+V_{2}+V_{3}$. Here we use the usual spectator notation where $V_{i}$ is the potential describing the interaction of particles $j$ and $k$ with particle $i$ being a spectator ( $i j k$ is a cyclic permutation of 123). Explicitly we have that

$$
V_{i}=v_{i} d_{i}^{-1}
$$

where $v_{i}$ is the two-body potential. As $V^{\mu}=V_{1}^{\mu}+V_{2}^{\mu}+V_{3}^{\mu}$, to obtain $V^{\mu}$ it is sufficient to gauge Eq. (7). To gauge the inverse propagator $d^{-1}$ we formally gauge the identity $d^{-1} d=1$, in this way obtaining $\left(d^{-1}\right)^{\mu}=-d^{-1} d^{\mu} d^{-1}=-\Gamma^{\mu}$. Thus $V_{i}^{\mu}=v_{i}^{\mu} d_{i}^{-1}-v_{i} \Gamma_{i}^{\mu}$. The negative sign in front of the term $v_{i} \Gamma_{i}^{\mu}$ may appear to be surprising, yet it is just what is needed to stop overcounting. Consider, for example, the gauging of the term $G_{0} V G$ appearing in Eq. (1): $\left(G_{0} V G\right)^{\mu}=G_{0}^{\mu} V G+G_{0} V^{\mu} G+G_{0} V G^{\mu}$. It is apparent that $v_{i} \Gamma_{i}^{\mu}$ appears in each of the three terms $G_{0}^{\mu} V G, G_{0} V^{\mu} G$, and $G_{0} V G^{\mu}$. Thus the negative sign in question is needed to ensure that $v_{i} \Gamma_{i}^{\mu}$ contributes only once to the gauging of $G_{0} V G$. The electromagnetic vertex function for the three-particle system is therefore

$$
\Gamma^{\mu}=\sum_{i=1}^{3}\left(\Gamma_{i}^{\mu} D_{0 i}^{-1}+v_{i}^{\mu} d_{i}^{-1}-v_{i} \Gamma_{i}^{\mu}\right)
$$

which we illustrate in Fig. 1. It is interesting to note that had one written the expression for $\Gamma^{\mu}$ by analogy to what is done in standard non-relativistic theory, one would get the wrong sign for $v_{i} \Gamma_{i}^{\mu}$. Just this was done in the pioneering four-dimensional calculations of the $N N N$ system by Rupp and Tjon [7]; as a result, this work contains overcounting.

\section{B. Gauging the AGS equations}

Although one can obtain the electromagnetic transition amplitudes for any process by taking appropriate bound state residues of $G^{\mu}$, for the three-body problem there exists an alternative approach that is especially convenient. This approach is based on the fourdimensional version of the Alt-Grassberger-Sandhas (AGS) equations [8] which describe the scattering of three strongly interacting hadrons. The AGS equations provide a direct way 
to calculate amplitudes for physical processes like $N-d$ elastic scattering or breakup. They also take as input two-body t-matrices $t_{i}$ rather than the two-body potentials $v_{i}$ which form the input of Eq. (1) for the Green function $G$. Thus by gauging the AGS equations one obtains electromagnetic transition amplitudes in a direct way, with the further advantage of having gauged t-matrices $t_{i}^{\mu}$ as input rather than gauged potentials $v_{i}^{\mu}$ which form the input of $G^{\mu}$.

For distinguishable particles the AGS equations can be written in the matrix form

$$
\tilde{U}=\mathcal{I} G_{0}+\mathcal{I} G_{0} T \tilde{U} \text {. }
$$

Here $\tilde{U}$ is a matrix whose the $(i, j)^{\prime}$ th element is defined by $\tilde{U}_{i j}=G_{0} U_{i j} G_{0}$ where $U_{i j}$ is the usual AGS amplitude describing the the scattering of particle $j$ off the $(k i)$ quasi-particle leading to a final state consisting of particle $i$ and the $(j k)$ quasi-particle. The other matrix quantities in Eq. (9) are defined by $T_{i k}=\delta_{i k} t_{k} d_{k}^{-1}$ and $\mathcal{I}_{i k}=1-\delta_{i k}$. Eq. (9) may now be gauged in just the same way as Eq. (11). We obtain that

$$
\tilde{U}^{\mu}=\tilde{U} \Gamma^{\mu} \tilde{U}
$$

where $\Gamma^{\mu}$ is a matrix whose $(n, m)$ 'th element is

$$
\Gamma_{n m}^{\mu}=\mathcal{I}_{n m}^{-1} \sum_{i=1}^{3} \Gamma_{i}^{\mu} D_{0 i}^{-1}+\delta_{n m} t_{n}^{\mu} d_{n}^{-1}-\delta_{n m} t_{n} \Gamma_{n}^{\mu} .
$$

This result can now be used to calculate electromagnetic processes of three-body systems. For example, to calculate $j(k i) \rightarrow i(j k) \gamma$ where $(k i)$ and $(j k)$ represent two-body bound states with bound state vertex functions $\phi_{j}$ and $\phi_{i}$ respectively, we first write the physical t-matrix for the process $j(k i) \rightarrow i(j k)$ as $T_{i j}=d_{i}^{-1} \bar{\phi}_{i} \tilde{U}_{i j} \phi_{j} d_{j}^{-1}$, and then gauge $T_{i j}$ using the product rule.

\section{Application to the gauged three-nucleon system}

For identical particles there is a variety of ways to define the AGS amplitude, all giving the same three-body Green function $G$. The one we have chosen, which we call $Z$, satisfies the particularly simple AGS equation for identical particles

$$
\tilde{Z}=G_{0}+D_{03} t_{3} \mathcal{P} \tilde{Z}
$$

where $\tilde{Z}=G_{0} Z G_{0}$ and where $\mathcal{P}$ shifts particle labels cyclically to the left. As $\tilde{Z}$ is related to the three-body Green function $G$ in a simple way, the solution to our problem of specifying $G^{\mu}$ now rests essentially on the construction of the gauged AGS Green function $\tilde{Z}^{\mu}$. Gauging Eq. (12) in the same way we gauged Eq. (11), we obtain

$$
\tilde{Z}^{\mu}=\tilde{Z} d_{3}^{-1} d_{3}^{\mu}+\tilde{Z}\left(D_{03}^{-1} D_{03}^{\mu} D_{03}^{-1} d_{3}^{-1}+d_{3}^{-1} t_{3}^{\mu} \mathcal{P}\right) \tilde{Z} .
$$

This equation describes the attachment of photons at all possible places in the multiplescattering series of three identical particles. The input is in terms of two-body t-matrices. As such, it forms the central result in the gauged three-nucleon problem. 
[1] A. N. Kvinikhidze and B. Blankleider, in preparation.

[2] A. Kvinikhidze and B. Blankleider, nucl-th/9706051, to be published in Phys. Rev. C. (1997).

[3] A. Kvinikhidze and B. Blankleider, nucl-th/9706052, to be published in Phys. Rev. C. (1997).

[4] J. C. Ward, Phys. Rev. 78 (1950) 182; Y. Takahashi, Nuovo Cimento 6 (1957) 371.

[5] W. Bentz, Nucl. Phys. A446 (1985) 678.

[6] F. Gross and D. O. Riska, Phys. Rev. C 36 (1987) 1928.

[7] G. Rupp and J. A. Tjon, Phys. Rev. C 37 (1988) 1729; ibid. 45 (1992) 2133.

[8] E. O. Alt, P. Grassberger, and W. Sandhas, Nucl. Phys. B2 (1967) 167. 